\title{
Small Angle Neutron Scattering Observation of Chain Retraction after a Large Step Deformation
}

\author{
A. Blanchard, ${ }^{1}$ R. S. Graham, ${ }^{2}$ M. Heinrich, ${ }^{1}$ W. Pyckhout-Hintzen, ${ }^{1}$ and D. Richter ${ }^{1}$ \\ ${ }^{1}$ Forschungszentrum Jülich, Institut für Festkörperforschung, D-52425 Jülich, Germany \\ ${ }^{2}$ Department of Chemical Engineering, University of Michigan, Ann Arbor, Michigan 48109, USA
}

\author{
A.E. Likhtman, ${ }^{3}$ T. C. B. McLeish, ${ }^{3}$ and D. J. Read ${ }^{4}$ \\ ${ }^{3}$ Department of Physics and Astronomy, University of Leeds, Leeds LS2 9JT, United Kingdom \\ ${ }^{4}$ Department of Applied Mathematics, University of Leeds, Leeds LS2 9JT, United Kingdom \\ E. Straube ${ }^{5}$ and J. Kohlbrecher ${ }^{6}$ \\ ${ }^{5}$ Martin-Luther-Universität Halle-Wittenberg, Fachbereich Physik, D-06099 Halle, Germany \\ ${ }^{6}$ Abteilung Spallationsquelle, Paul Scherrer Institut, $\mathrm{CH}-5232$ Villigen, Switzerland
}

(Received 18 November 2004; published 14 October 2005)

\begin{abstract}
The process of retraction in entangled linear chains after a fast nonlinear stretch was detected from time-resolved but quenched small angle neutron scattering (SANS) experiments on long, well-entangled polyisoprene chains. The statically obtained SANS data cover the relevant time regime for retraction, and they provide a direct, microscopic verification of this nonlinear process as predicted by the tube model. Clear, quantitative agreement is found with recent theories of contour length fluctuations and convective constraint release, using parameters obtained mainly from linear rheology. The theory captures the full range of scattering vectors once the crossover to fluctuations on length scales below the tube diameter is accounted for.
\end{abstract}

DOI: 10.1103/PhysRevLett.95.166001

PACS numbers: 83.10.Kn, 61.12.Ex, 83.60.Df

Both fundamental and applied understanding of polymer dynamics through the use of molecular based theories has seen rapid progress in the past 20 years [1]. This progress stems from the realization that surrounding chains severely limit global motion of a test chain in directions perpendicular to its contour but do not prohibit motion along it, confining each chain to a tubelike region defined by its own contour [2]. This physical picture is beautifully cast into a physical theory by the reptation model of de Gennes [3]. The current challenge is to augment this concept of onedimensional diffusion of a chain along a tube into a quantitative molecular approach describing both the linear and nonlinear rheological properties as well as the underlying molecular motions [4-6].

One important question in this context relates to the chain relaxation in elongational flow. A large step extensional elongation is expected to affinely deform the chain contour. This will cause the chain radius of gyration parallel to the flow to increase and conversely the one in the perpendicular direction to decrease. After cessation of the strain, one expects the first relaxation process to be a retraction of the chain within the still affinely deformed tube. The longest time scale of this process should be the equilibration time $\tau_{\mathrm{R}}$ (Rouse time) of the chain along the tube. This mechanism is related by a fluctuationdissipation theorem to the chain contour length fluctuations, entropically driven extension and retraction of the chain contour which recently was corroborated directly on a molecular level [7].

Chain retraction in the elongated tube should reduce the radius of gyration in all directions. Therefore $R_{g}^{\text {perp }}$ is expected to attain a minimum, manifested as an increase in perpendicular scattered intensity, at some time after the deformation determined by the Rouse time, before diffusive mechanisms return it to equilibrium. This nonmonotonic behavior, with the minimum at a time consistent with the time scale of Rouse chain retraction, was not observed in previous experiments on polystyrene $[8,9]$ and poly(ethylethylene) melts [10]. The requirement of a measurable Rouse time demanded prohibitively large overall chain dimensions so observations were possible only along the perpendicular axis of the stretch. Moreover, the data analysis remained speculative as no quantitative tube approach was available at the time [11-17].

In this Letter we present a successful new approach to observe the basic nonlinear relaxation mechanism of chain retraction. We use a new deformation device, suitable for investigation of low $T_{g}$ materials with in situ small angle neutron scattering (SANS) [18], to study flexible chains with narrow tube dimensions and accurately established relaxation times. The investigated polyisoprene sample has length and time scales specifically tailored to the experimental conditions. Furthermore, we use recent theoretical improvements, which describe the full gamut of relaxation processes, to facilitate a proper evaluation of the data. Our results unequivocally demonstrate the so far elusive nonlinear relaxation process, providing another important confirmation of the tube concept in nonlinear rheology.

Protonated and deuterated polyisoprene homopolymers were synthesized by anionic polymerization in $n$-hexane under high vacuum with sec-Butyllithium as initiator. Virtually identical molecular weights, with respect to de- 
gree of polymerization, were obtained ( $d$-PI: $M_{w}=$ $254000 \mathrm{~g} / \mathrm{mol}, \quad M_{w} / M_{n}=1.019 ; \quad h$-PI: $\quad M_{w}=$ $\left.240000 \mathrm{~g} / \mathrm{mol}, M_{w} / M_{n}=1.020\right)$ and solution blended in a ratio of $90 / 10 h / d$ by mass. The $h$-PI was measured in $1 \%$ linear shear on an ARES rheometer between $10^{-2}-10^{2} \mathrm{rad} \cdot \mathrm{s}^{-1}$ and temperatures between -35 and $55^{\circ} \mathrm{C}$.

SANS experiments were carried out at SANS-1, PSI, CH at a wavelength $\lambda$ of $0.8 \mathrm{~nm}$ to cover a scattering vector range $0.03 \leq q \leq 2 \mathrm{~nm}^{-1}$. The data were corrected for empty beam, detector sensitivity, solid angle dependency, and absolutely calibrated with a $1 \mathrm{~mm}$ water standard. Radial, as well as sector, averaging in the anisotropic case with opening angle $10^{\circ}$ was used to allow principal axes to be analyzed.

The samples were mounted in a homebuilt elongational device [18] at $-45^{\circ} \mathrm{C}$ in $N_{2}$ atmosphere and uniaxially stretched to a strain of $\lambda=1.7$ in $25 \mathrm{~s}$ at a constant strain rate corresponding to $2300 \mathrm{~s}^{-1}$ at $\mathrm{RT}\left(\dot{\epsilon} \tau_{\mathrm{R}} \approx 120\right)$. Microscopic relaxation times and sampling time were decoupled by an appropriate quench to $T<T_{g}$ within $1 \mathrm{~s}$. The initial deformed state was measured as the reference. Relaxation times of $0.4,3.22,8.44$, and 15.72 times $\tau_{\mathrm{R}}(0.045 \mathrm{~s}$ at RT) were achieved by annealing the sample gradually through a temperature ramp for which equivalent times at RT are calculated using known linear timetemperature shifts, then quenching anew.

Experimental plots of scattered intensity against scattering vector (Fig. 1) demonstrate that, throughout the relaxation regime, the anisotropy is strong at large length scales, weakening as the scale of the tube diameter is reached at higher $q$. As relaxation progresses, so features at higher $q$ relax to isotropy faster than at lower $q$. At $t=3.22 \tau_{\mathrm{R}}$ the parallel scattering shows significant relaxation relative to the fully affine curves (dotted lines). However, throughout this time interval, the perpendicular scattering remains close to the affine curve over almost the entire range of scattering vectors [Fig. 1(a)-1(c)]. Of especial note is the small, but significant, increase in scattered intensity between $t=0$ and $0.4 \tau_{\mathrm{R}}$, visible in the perpendicular component of the scattering [Fig. 2(c)]. We take this to be an unambiguous experimental signature of chain retraction, while relying on the comparison with theory to confirm that the magnitude of the increase is consistent with the retraction process.

In the model of Graham et al. [5] the relaxation mechanisms of reptation, contour length fluctuations, retraction, and constraint release are formulated into a stochastic microscopic evolution equation for the dynamics of the space curve describing the tube contour, $\mathbf{R}_{s}(t)$, where $s$ is a continuous variable labeling distance along the tube contour and $t$ is time. From this equation a deterministic partial differential equation for the tube tangent vector correlation function, $\mathbf{f}\left(s, s^{\prime}, t\right)=\left\langle\frac{\partial \mathbf{R}_{s}(t)}{\partial s} \frac{\partial \mathbf{R}_{s^{\prime}}(t)}{\partial s^{\prime}}\right\rangle$, is derived, allowing the tube configuration, stress tensor, and the single chain structure factor to be computed for any nonlinear deformation. For full details see Refs. [5,19].

Following exactly the approach in Ref. [5] the two material-dependent parameters were determined by fitting the linear theory of Likhtman and McLeish [4] to linear oscillatory shear data, yielding RT values of $\tau_{e}=1.34 \times$ $10^{-5} \mathrm{~s}$ and $G_{e}=5.05 \times 10^{5} \mathrm{~Pa}$, which indicates that the chains have 58 entanglements and a Rouse time of $0.045 \mathrm{~s}$. The chain radius of gyration was obtained by fitting a Debye function to scattering data from the fully relaxed melt, then the relation $R_{g}^{2}=a^{2} Z / 6$ leads to a tube diameter value of $a=6.3 \mathrm{~nm}$. Note that, since the degree of entanglement, $Z$, is determined by the entanglement modulus, $G_{e}$, the value of the tube diameter and therefore the tube persistence length are deduced from rheological measurements alone.

The theory outlined in [5] predicts the shape of the coarse-grained tube path, which is sufficient to predict changes in $R_{g}$. However, it provides no detail on length scales shorter than the tube diameter, necessary for a data (a)
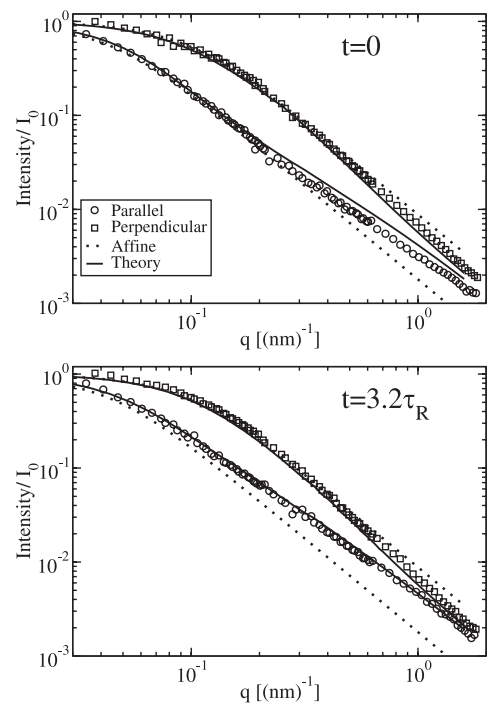

(b)

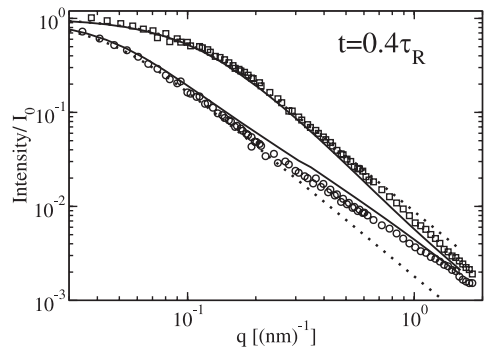

(d)

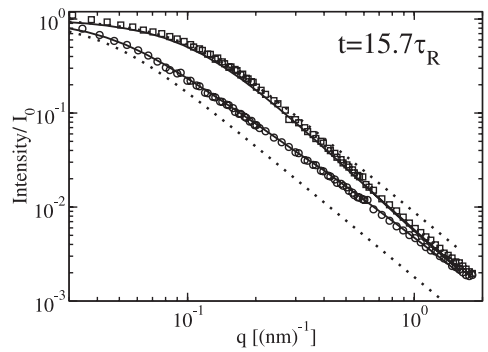

FIG. 1. Comparison of measured and predicted normalized structure factors after $0,0.4 \tau_{\mathrm{R}}, 3.2 \tau_{\mathrm{R}}$, and $15.7 \tau_{\mathrm{R}}[(\mathrm{a})-$ (d), respectively]. The solid lines are predictions of the theory of Graham et al. [5], and the dotted lines correspond to a completely affine deformation on all length scales. 

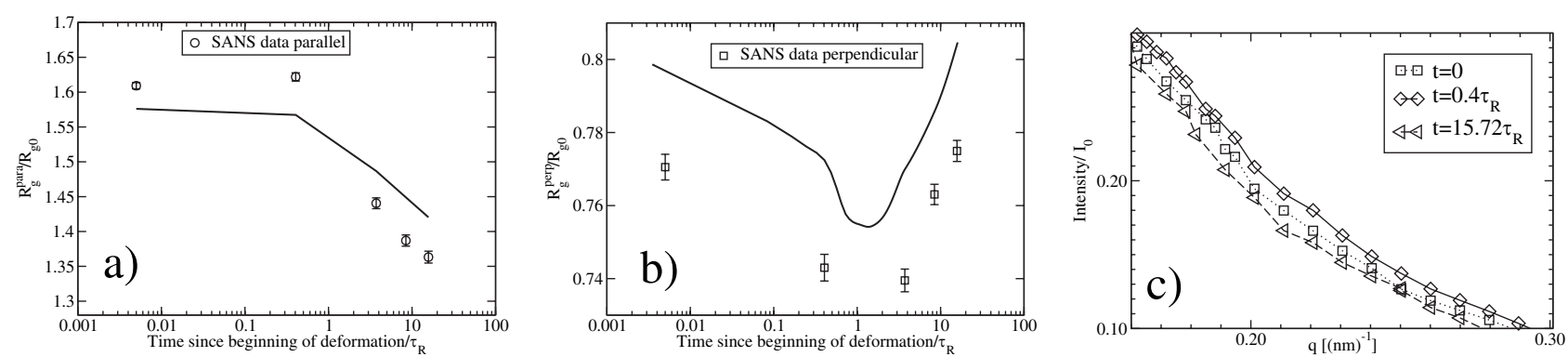

FIG. 2. Data and theory for relaxation of the radius of gyration parallel and perpendicular to the stretch direction after deformation (a),(b) and experimental data highlighting the nonmonotonic region of $R_{g}^{\text {perp }}$ (c).

comparison across the measured range of wave vectors. In particular, the high- $q$ scattering is dominated by chain fluctuations about the mean path defined by the tube, for which we need a suitable crossover formula. The structure factor can be expressed in terms of the monomer positions, $\mathbf{r}_{s}$,

$$
S(\mathbf{q})=\frac{1}{Z^{2}} \int_{0}^{Z} \int_{0}^{Z} \exp \left(-\sum_{\alpha, \beta} \frac{q_{\alpha} q_{\beta}}{2} \overline{\left\langle\left(r_{\alpha s}-r_{\alpha s^{\prime}}\right)\left(r_{\beta s}-r_{\beta s^{\prime}}\right)\right\rangle}\right) d s d s^{\prime}
$$

where $\overline{(\cdots)}$ and $\langle\cdots\rangle$ denote averages over monomer fluctuations and tube positions, respectively, and $\alpha$ and $\beta$ are Cartesian components. Therefore we require an expression for the following two-point monomer correlation

$$
\overline{\left\langle\left(r_{\alpha s}-r_{\alpha s^{\prime}}\right)\left(r_{\beta s}-r_{\beta s^{\prime}}\right)\right\rangle},
$$

which must be valid for all length scales probed by the scattering experiment. The mean position of each monomer, $\mathbf{R}$, is defined by the tube, and we denote the fluctuations about this mean by $\boldsymbol{\Delta}_{s}$.

$$
r_{\alpha s}=R_{\alpha s}+\Delta_{\alpha s}
$$

To obtain a straightforward, but usable, crossover formula, we make the simplest possible assumption about the mono- mer fluctuations, $\Delta_{s}$ : we assume these are statistically isotropic, with a magnitude independent of deformation of the mean position, $\mathbf{R}_{s}$, allowing us to write

$$
\begin{aligned}
\overline{\left\langle\left(r_{\alpha s}-r_{\alpha s^{\prime}}\right)\left(r_{\beta s}-r_{\beta s^{\prime}}\right)\right\rangle}= & \left\langle\left(R_{\alpha s}-R_{\alpha s^{\prime}}\right)\left(R_{\beta s}-R_{\beta s^{\prime}}\right)\right\rangle \\
& +\overline{\left(\Delta_{\alpha s}-\Delta_{\alpha s^{\prime}}\right)\left(\Delta_{\beta s}-\Delta_{\beta s^{\prime}}\right) .}
\end{aligned}
$$

The average over mean positions can be found by integrating the deformed tube tangent correlation function as computed by the theory

$$
\left\langle\left(R_{\alpha s}-R_{\alpha s^{\prime}}\right)\left(R_{\beta s}-R_{\beta s^{\prime}}\right)\right\rangle=\int_{s}^{s^{\prime}} \int_{s}^{s^{\prime}} f_{\alpha \beta}\left(s_{1}, s_{2}\right) d s_{1} d s_{2} .
$$

For the chains to obey Gaussian statistics in equilibrium the monomer fluctuations must be

$$
\overline{\left(\Delta_{\alpha s}-\Delta_{\alpha s^{\prime}}\right)\left(\Delta_{\beta s}-\Delta_{\beta s^{\prime}}\right)}=\frac{a^{2} \delta_{\alpha \beta}}{3}\left|s-s^{\prime}\right|-\int_{s}^{s^{\prime}} \int_{s}^{s^{\prime}} f_{\alpha \beta}^{\mathrm{eq}}\left(s_{1}, s_{2}\right) d s_{1} d s_{2},
$$

$f_{\alpha \beta}^{\mathrm{eq}}\left(s, s^{\prime}\right)$ is the equilibrium tube configuration, given by

$$
f_{\alpha \beta}^{\mathrm{eq}}\left(s, s^{\prime}\right)= \begin{cases}\frac{a^{2}}{3} \delta_{\alpha \beta} & \text { if }\left|s-s^{\prime}\right|<1 / 2 \\ 0 & \text { otherwise }\end{cases}
$$

in which tangent correlations persist for the length of a single tube segment. With the assumption that monomer fluctuations are independent of the deformation, Eq. (4) is valid for the deformed case as well. Adding these two contributions, we obtain an expression for the desired average monomer correlation that can be used in Eq. (1)

$$
\overline{\left\langle\left(r_{\alpha s}-r_{\alpha s^{\prime}}\right)\left(r_{\beta s}-r_{\beta s^{\prime}}\right)\right\rangle}=\frac{a^{2} \delta_{\alpha \beta}}{3}\left|s-s^{\prime}\right|+\int_{s}^{s^{\prime}} \int_{s}^{s^{\prime}}\left[f_{\alpha \beta}\left(s_{1}, s_{2}\right)-f_{\alpha \beta}^{\mathrm{eq}}\left(s_{1}, s_{2}\right)\right] d s_{1} d s_{2} .
$$

A similar expression to this is obtained from the WarnerEdwards [20] model for polymer networks, with the assumption of isotropic localizing potentials, which corresponds directly to our assumption of isotropic fluctuations [21]. The model predictions for the tube configuration were computed using a deformation history corresponding to that from the experiments. After each relaxation interval, the resulting tube configuration was used to compute the single chain structure factor via Eq. (1).
Figure 1 shows a comparison between data and theoretical predictions for the full range of scattering vectors at a range of relaxation intervals. The agreement along the principle strain axes between theory and experiment is close, especially considering the complete absence of nonlinear fitting parameters. The only marked region of disagreement occurs around $q a \gtrsim 1$ for the shortest relaxation intervals $\left(t<\tau_{\mathrm{R}}\right)$, and the disagreement lessens as $t$ increases further. There are several possible sources of the 
small discrepancies. First, deformation of the tube localizing potentials $[21,22]$ may occur in our experiments, but a treatment of this effect requires a new generation of entanglement models, which contain a more detailed description of constraints and their evolution. Second, the crossover function, used in Eq. (5), may be too crude and more elaborate choices can be made. Furthermore, some of the discrepancy may also be attributed to a recently reported inconsistency of the tube model, in which the tube diameter extracted from rheology is different from the value obtained from neutron spin echo or diffusion measurements $[23,24]$. In fact, fitting the data at $t=0$ with the Warner-Edwards model, where the tube diameter is a free fitting parameter, suggests a value $\approx 4 \mathrm{~nm}$. A tentative calculation with this smaller tube diameter relieves the disagreement in the location of the crossover to subtube length scales. However, our primary concern, evidence for retraction contained in the low- $q$ data, is not influenced by these details.

A plot of the relaxation of the deformed radius of gyration with time portrays the SANS signature of chain retraction (Fig. 2). Since an affine Debye function will not simultaneously capture the whole $q$ range of the deformed data, we obtained deformed radii by fitting a Debye function to the $q$ region roughly corresponding to the dimensions of the whole chain. The greatest sensitivity to this deformed $R_{g}$ occurs in the region around $q \sim 0.2 \mathrm{~nm}^{-1}$ so we used an unweighted least squares fitting procedure to emphasize this low- $q$ region. Values were acquired from the theory in an identical manner. Uncertainties in the deformed $R_{g}$ due to neutron counting errors were obtained by using the bootstrap method [25]. Figure 2(b) clearly shows the experimental observation of a pronounced minimum in $R_{g}^{\text {perp }}(t)$, providing a microscopic confirmation of the tube retraction concept. The minimum is not a random artifact due to counting errors since its depth significantly exceeds these error bars. The applied macroscopic strain is subject to an uncertainty of $\sim 5 \%$, which does not affect the observation of retraction since all data points are from a single deformation. The theory demonstrates that the time and depth of this minimum are consistent with the retraction process, although the model systematically underpredicts the degree of deformation by $\sim 4 \%$ of the total deformation. This shift can be explained by uncertainties in the experimentally applied strain. Figure 2(c) shows a magnified plot of experimental data for the perpendicular direction in the region of nonmonotonic behavior to further demonstrate the evidence for retraction.

In conclusion, we have tracked the relaxation of monodisperse, linear entangled polymers following a large uniaxial extension step using SANS measurements. These measurements elucidate chain relaxation over a broad range of relevant length scales and were compared to the predictions of a recent nonlinear tube model. Good quantitative agreement was found between data and theory over a wide range of length scales, including a minimum in the deformed radius of gyration perpendicular to the stretch, observed a short time after cessation of flow. This longstanding consequence of the retraction process had not, previously, been confirmed experimentally. Modeling these data provides a significant quantitative test for molecular theory and illustrates the possibility of linking molecular structure and processing history.

The authors thank M. Hintzen for the characterization of the polymers. Special thanks go to H. Feilbach and P. Eickenberg for the reconstruction of the furnace (IFFFZJ Workshop) and to the PSI for the availability of EU funding and allocation of urgent beam time.

[1] T. C. B. McLeish, Adv. Phys. 51, 1379 (2002).

[2] M. Doi and S.F. Edwards, The Theory of Polymer Dynamics (Oxford University Press, Oxford, 1986).

[3] P.-G. de Gennes, J. Chem. Phys. 55, 572 (1971).

[4] A. E. Likhtman and T. C. B. McLeish, Macromolecules 35, 6332 (2002).

[5] R. S. Graham, A. E. Likhtman, T. C. B. McLeish, and S. T. Milner, J. Rheol. (N.Y.) 47, 1171 (2003).

[6] J. Bent et al., Science 301, 1691 (2003).

[7] A. Wischnewski, M. Monkenbusch, L. Willner, D. Richter, A.E. Likhtman, T.C.B. McLeish, and B. Farago, Phys. Rev. Lett. 88, 058301 (2002).

[8] F. Boué, Adv. Polym. Sci. 82, 47 (1987).

[9] F. Boué, M. Nierlich, and K. Osaki, Faraday Symp. Chem. Soc. 18, 83 (1983).

[10] K. Mortensen, O. Kramer, W. Batsberg, and L. Fetters, Mater. Res. Soc. Symp. Proc. 79, 259 (1987).

[11] J. Viovy, J. Phys. (Paris) 46, 847 (1985).

[12] J. Viovy, J. Polym. Sci. 23, 2423 (1985).

[13] J. Viovy, J. Polym. Sci., Part B: Polym. Phys. 24, 1611 (1986).

[14] J. Viovy, L. Monnerie, and J. Tassin, J. Polym. Sci. 21, 2427 (1983).

[15] K. Hong and J. Noolandi, Makromol. Chem., Rapid Commun. 4, 617 (1983).

[16] M. Doi and S. Edwards, J. Chem. Soc., Faraday Trans. 2 74, 1802 (1978).

[17] R. Muller, C. Picot, Y. Zang, and D. Froelich, Macromolecules 23, 2577 (1990).

[18] M. Heinrich, W. Pyckhout-Hintzen, J. Allgaier, D. Richter, E. Straube, D. J. Read, T. C. B. McLeish, D. J. Groves, R. J. Blackwell, and A. Wiedenmann, Macromolecules 35, 6650 (2002)

[19] R. S. Graham, Ph.D. thesis, University of Leeds, 2002.

[20] M. Warner and S. F. Edwards, J. Phys. A 11, 1649 (1978).

[21] D. J. Read, Macromolecules 37, 5065 (2004).

[22] M. Rubinstein and S. Panyukov, Macromolecules 35, 6670 (2002).

[23] J. T. Padding and W. J. Briels, J. Chem. Phys. 120, 2996 (2004).

[24] K. Kremer, in Jülich Soft Matter Days (Cambridge University Press, Cambridge, England, 2003).

[25] W. H. Press, B.P. Flannery, S. A. Teukolsky, and W. T. Vetterling, Numerical Recipes in C: The Art of Scientific Computing (Cambridge University Press, Cambridge, England, 1992). 